\title{
COGNIÇÕES MATERNAS ACERCA DA MATERNIDADE E DO DESENVOLVIMENTO HUMANO: UMA CONTRIBUIÇÃO AO ESTUDO DA PSICOLOGIA PARENTAL
}

\author{
MOTHERS' COGNITIONS ABOUT MOTHERING AND HUMAN \\ DEVELOPMENT: A CONTRIBUTION TO THE STUDY OF PARENTAL \\ PSYCHOLOGY
}

\begin{abstract}
Ribas Jr RdC, Moura MLSd, Bornstein MH. Cognições maternas acerca da maternidade e do desenvolvimento humano: Uma contribuição ao estudo da psicologia parental. Rev Bras Crescimento Desenvolv Hum 2007; 17(1):104-113.
\end{abstract}

\begin{abstract}
Resumo: O presente estudo investigou três tipos de cognições parentais (conhecimentos acerca do desenvolvimento infantil e da criação de filhos, autopercepções parentais e atribuições de causalidade parentais) e teve três objetivos: 1) ampliar nosso conhecimento sobre cognições de mães brasileiras acerca da maternidade, 2) comparar resultados da presente investigação com os de estudos anteriores e 3) investigar associações entre as cognições maternas e entre essas cognições e determinadas variáveis sociodemográficas. Ao todo, 66 mães primíparas, com idades acima de 18 anos, de famílias intactas, residentes na cidade do Rio de Janeiro participaram do estudo. As mães responderam a versões brasileiras de quatro instrumentos (Inventário de Conhecimento do Desenvolvimento Infantil, Autopercepção do Papel Parental, Questionário de Atribuições Parentais, Escala Marlowe-Crowne de Desejabilidade Social) e um questionário sociodemográfico. As mães responderam corretamente a $63 \%$ das questões do instrumento que avaliou conhecimento parental, indicando que seu conhecimento sobre o desenvolvimento infantil pode ser ampliado. Em termos de autopercepções, os aspectos mais positivamente avaliados pelas mães foram a satisfação e investimento no papel parental. As mães tenderam a avaliar as causas externas (e.g., comportamento do filho) como mais importantes do que as internas (e.g., esforço), tanto para o sucesso quanto para o fracasso nas atividades parentais. Várias associações entre as cognições maternas e delas com variáveis sociodemográficas (e.g., educação) foram identificadas. Os resultados foram, em larga medida, consistentes com estudos nacionais e internacionais anteriores e podem contribuir para o entendimento das cognições parentais como um sistema complexo.
\end{abstract}

Palavras chave: Cognições parentais. Conhecimentos. Autopercepções. Atribuições causais.

O presente trabalho, que focaliza cognições maternas acerca de temas relacionados ao desenvolvimento humano e à maternidade, apresenta resultados parciais de projeto de pesquisa longitudinal e transcultural mais amplo ${ }^{1,2}$.
Diversos motivos têm sido apresentados para justificar a investigação da cognição parental. Pode-se argumentar, por exemplo, que um maior conhecimento de cognições sociais relacionadas à criação de filhos contribui para um melhor

Universidade Federal do Rio de Janeiro. Endereço para correspondência: Rodolfo de Castro Ribas Jr., Rua Maria Amália. 87/401, Tijuca, Rio de Janeiro, Brasil. CEP 20510-130. E-mail: rribas@ufrj.br

A preparação deste artigo foi, em parte, financiada pela Jacobs Foundation, Zurique, Suíça, por meio de uma bolsa (Dissertation Grant) concedida ao primeiro autor.

** Universidade do Estado do Rio de Janeiro

*** National Institute of Child Health and Human Development 
entendimento de um importante aspecto da vida adulta, a criação de filhos. Por outro lado, pesquisas têm indicado que as cognições parentais acerca do desenvolvimento humano e da paternidade influenciam a forma como pais e mães se relacionam com seus filhos e com crianças de modo geral, e que esses comportamentos, por sua vez, influenciam o próprio desenvolvimento infantil. Assim, um melhor entendimento das cognições parentais contribuiria para um melhor entendimento do próprio desenvolvimento infantil ${ }^{3}$.

Basicamente, três objetivos orientaram este trabalho. O primeiro foi ampliar o nosso conhecimento sobre cognições que mães brasileiras têm acerca do desenvolvimento infantil e da tarefa de cuidar dos filhos. Diferentes tipos de cognições conhecimentos parentais, autopercepções parentais e atribuições de causalidade para o sucesso e o fracasso nas atividades parentais - foram investigados simultaneamente. O segundo objetivo foi comparar os resultados obtidos neste estudo aos produzidos em estudos anteriores com metodologia similar. Finalmente, foram investigadas associações entre as diferentes cognições e entre estas e determinadas variáveis sociodemográficas (e.g., idade, escolaridade e status socioeconômico).

\section{Conhecimentos acerca do desenvolvimento infantil e da criação de filhos}

Os conhecimentos parentais são hoje um dos mais investigados temas de pesquisa relativos à cognição parental. Conhecimentos parentais vêm sendo definidos como crenças acerca da realidade que, em alguma medida, são endossadas por membros da comunidade científica ${ }^{4}$. Por exemplo: "o leite materno é normalmente o melhor alimento para os bebês recém-nascidos".

Diversos trabalhos têm apontado que conhecimentos parentais são continuamente mobilizados pelos pais para avaliar o comportamento e o desenvolvimento de seus filhos e para guiar decisões cotidianas a respeito de como conduzir a criação de filhos. Benasich e Brooks-Gunn ${ }^{5}$, por exemplo, verificaram que o nível de conhecimento materno se mostrou um bom preditor da qualidade do ambiente que as mães ofereciam para os filhos em casa, mesmo quando o status socioeconômico da família foi controlado. $\mathrm{O}$ estudo, que envolveu mães adultas e adolescentes, indicou ainda que mães adultas e com maior escolaridade tendem a ter mais conhecimento acerca do desenvolvimento infantil.

Estudos acerca de conhecimentos parentais sobre a saúde e a segurança física de crianças têm fornecido informações extremamente úteis para governos e agências de saúde. Angelillo et al. ${ }^{6}$, por exemplo, estudaram atitudes de mães a respeito da vacinação infantil e indicaram que a falta de conhecimento estava impedindo que mães italianas desempenhassem um importante papel na erradicação de doenças evitáveis com vacinas na Itália.

\section{Atribuições parentais de causalidade}

O estudo das atribuições parentais de causalidade caracteriza outra destacada área de pesquisa sobre a cognição parental. A idéia que fundamenta as pesquisas sobre atribuições parentais de causalidade é a de que pais constroem teorias acerca dos determinantes dos comportamentos de seus filhos e dos seus próprios comportamentos. Evidências têm consistentemente indicado que atribuições parentais para o sucesso e o fracasso no exercício do papel parental são importantes preditores de práticas parentais consideradas mais efetivas ${ }^{7}$. Pesquisas têm revelado, por exemplo, que pais que superestimam a influência de fatores fora de seu próprio controle tendem a apresentar estilos parentais mais coercitivos ${ }^{8}$.

Stevens ${ }^{9}$ investigou atribuições de causalidade e expectativas acerca do desenvolvimento e verificou, entre outros aspectos, que mães que apresentaram um lócus de controle externo para o sucesso (i.e., que apontaram a sorte, a fatalidade e outras pessoas como os determinantes mais importantes para eventos em suas vidas) tenderam a apresentar conhecimento menos acurado a respeito do desenvolvimento infantil.

\section{Autopercepções parentais}

O conceito de autopercepção parental refere-se, basicamente, à maneira como os pais se percebem no desempenho do papel parental. As autopercepções parentais podem ser enten- 
didas como autoconceitos, ou seja, crenças e atitudes que indivíduos têm acerca de si mesmos. Entretanto, mais freqüentemente as autopercepções parentais vêm sendo associadas ao conceito de auto-eficácia ${ }^{10}$ e têm sido avaliadas como a maneira pela qual os pais percebem a própria eficácia no desempenho no papel parental. De acordo com Coleman e Karraker ${ }^{7}$, evidências têm consistentemente indicado que crenças parentais acerca da auto-eficácia são poderosos preditores de práticas parentais consideradas mais efetivas e mediadores dos efeitos de algumas das variáveis que mais usualmente têm sido associadas à qualidade das práticas parentais (e.g., depressão materna, temperamento infantil, suporte social e status socioeconômico).

Ruchala e James ${ }^{11}$ investigaram as relações entre conhecimento sobre o desenvolvimento infantil, percepção da auto-eficácia e suporte social em um grupo de mães. Mães com maior conhecimento acerca do desenvolvimento infantil apresentaram uma percepção mais positiva de seu desempenho no papel parental. Em um recente estudo brasileiro ${ }^{12}$ foi observado, entre outros aspectos, que pais que possuíam uma melhor autopercepção de seu desempenho do papel parental tenderam a ter relações mais positivas e menos ásperas com seus filhos.

\section{Status socioeconômico, escolaridade e cognição parental}

Diversos trabalhos recentes têm ressaltado a relevância de variáveis como o status socioeconômico e a escolaridade para o entendimento das cognições e práticas parentais ${ }^{13}$. Um estudo recente envolvendo mães de diversas capitais brasileiras revelou uma correlação direta entre a escolaridade, status socioeconômico e o conhecimento de mães sobre o desenvolvimento infantil ${ }^{14}$. Outros estudos têm ainda sugerido, por exemplo, que famílias com diferentes níveis socioeconômicos podem ter diferentes sistemas de crenças e valores parentais ${ }^{15}$.

A realização desta pesquisa mostrou-se relevante sob vários aspectos. Conforme assinalado, conhecimentos, autopercepções e atribuições de causalidade parentais vêm sendo apon- tados como importantes preditores do comportamento parental. Uma revisão sistemática da literatura nacional pertinente revelou que estudos brasileiros sobre os tipos de cognições parentais estudadas no presente trabalho são ainda relativamente raros, embora tenha sido percebido um crescimento de publicações a partir da última década ${ }^{16}$. Vale também ressaltar que estudos sobre relações entre diferentes tipos de cognições parentais são ainda mais escassas, mesmo na literatura internacional.

Considerando esse contexto, entende-se que o presente estudo pode ampliar o nosso conhecimento sobre cognições que mães brasileiras em áreas ainda relativamente pouco estudadas e oferecer indicações acerca de associações entre essas diferentes cognições. Ao comparar os resultados obtidos neste estudo com resultados obtidos em estudos semelhantes realizados no Brasil e em outros países, pretendemos ainda oferecer informações que permitiam situar os nossos achados dentro de um contexto mais amplo.

\section{MÉTODO}

\section{Participantes}

Ao todo, 66 mães que residiam na região metropolitana da cidade do Rio de Janeiro participaram da pesquisa. Os critérios de inclusão no estudo foram os seguintes: mães primíparas, com idade igual ou superior a 18 anos e oriundas de famílias intactas (constituídas de marido e mulher, não necessariamente casados legalmente, que viviam sob o mesmo teto). As mães deveriam ter filhos naturais com aproximadamente 5 meses, nascidos a termo, sem problemas médicos ou psicológicos conhecidos. A técnica da bola de neve foi utilizada para a identificação e contato inicial com as mães que participaram da pesquisa. $\mathrm{O}$ processo de amostragem foi planejado de forma a obter mães com diferentes níveis de escolaridade e status socioeconômico, e um número aproximadamente igual de mães de meninas e mães de meninos.

Das 66 mães, apenas seis informaram ter participado de algum tipo de curso destinado a 
gestantes ou a pais. Dessas seis, quatro informaram que a participação em um curso não ajudou ou ajudou de forma limitada no desempenho do papel maternal. Somente duas informaram que a participação em cursos ajudou mais significativamente a desempenhar esse papel. Atividade profissional remunerada era realizada por $48,5 \%$ das mães. A Tabela 1 apresenta dados sociodemográficos das mães e de seus bebês.

Tabela 1 - Dados sociodemográficos das mães

\begin{tabular}{|lll|}
\hline $\mathrm{N}$ & 66 & \\
\hline Idade (anos) & 26,4 & $(5,7)$ \\
\hline Escolaridade $^{\mathrm{a}}$ & 4,39 & $(1,89)$ \\
\hline Status Socioeconômico $^{\mathrm{b}}$ & 42,4 & $(16,1)$ \\
\hline Status Ocupacional (\%) & & \\
Com atividade remunerada & 48,5 & \\
Sem atividade remunerada & 51,5 & $(0,2)$ \\
\hline Idade dos bebês (meses) & 5,0 & \\
\hline Sexo dos bebês (\%) & & \\
Meninas & 52,3 & \\
Meninos & 47,7 & \\
\hline
\end{tabular}

\section{Instrumentos}

Conhecimento do Desenvolvimento Infantil. Foi utilizada uma versão em português do Inventário de Conhecimento do Desenvolvimento Infantil (KIDI) ${ }^{14,17}$. O KIDI abrange 75 questões (e.g., "Em geral, os bebês não podem ver e ouvir ao nascerem".).que avaliam conhecimentos sobre a aquisição de habilidades motoras, perceptuais e cognitivas, fatores relacionados ao desenvolvimento e práticas parentais de alimentação, higiene e segurança. O escore no inventário é obtido dividindo-se o número de questões respondidas corretamente pelo número total de questões respondidas. Dessa forma, o inventário produz um escore que vai de 0 (pouco conhecimento) a 1 (muito conhecimento). No presente estudo foi observado um alfa de Cronbach de 0,78.

Autopercepção do Papel Parental. Foi empregada a versão em português do instrumento de avaliação da Autopercepção do Papel Parental $(\mathrm{SPPR})^{18,19}$, para avaliar a percepção das mães acerca de seu próprio desempenho no papel parental em quatro áreas: Competência, Satisfação, Investimento e Integração com outros papéis sociais de adultas (e.g., trabalhadora, esposa, amiga). Cada um dos 22 itens do questionário é constituído de duas afirmações contrastantes acerca da dimensão que se pretende avaliar (e.g., "Alguns pais acham que estão atendendo bem às necessidades de seus filhos", mas "Outros pais têm dúvidas se estão atendendo bem às necessidades de seus filhos"). O escore para cada subescala (que pode ter cinco ou seis itens) é obtido pela média simples das respostas daquela subescala e pode variar de 1 a 5 (valores mais altos indicam uma autopercepção mais positiva). No presente estudo foram obtidos os seguintes alfas que variaram de 0,49 a 0,60 .

Atribuições para o comportamento parental. Foi empregada uma versão em português do Questionário sobre Atribuições Parentais (PAQ) $)^{19,20}$, para avaliar as atribuições de causalidade para o sucesso e o fracasso em atividades de criação de filhos. O PAQ apresenta cinco tipos de atribuições de causalidade (habilidade da mãe, esforço da mãe, estado de humor da mãe, dificuldade da tarefa e comportamento da criança) que explicariam o sucesso ou o fracasso em sete tarefas parentais (vestir, dar banho, confortar, ensinar, disciplinar, comunicar e brincar). Cada uma das cinco atribuições é avaliada em escalas do tipo Likert (Nunca é uma razão, Dificilmente é uma razão, Em parte é uma razão, Certamente é uma razão, Com toda a certeza é uma razão). O PAQ pode produzir quatro subescalas: Atribuição Interna para o Sucesso e Atribuição Interna para o Fracasso, que incluiriam os itens relativos a três tipos de atribuições de causalidade (habilidade, esforço e estado de humor), e Atribuição Externa para o Sucesso e Atribuição Externa para o Fracasso, que incluiriam os itens relativos a dois tipos de atribuições de causalidade (dificuldade da tarefa e comportamento da criança). Essas subescalas oferecem indicadores de lócus de controle parental. No presente estudo os alfas para as quatro subescalas oscilaram entre 0,81 e 0,88 .

Desejabilidade Social. Uma versão em português da Escala de Desejabilidade Social de Marlowe-Crowne (MC-SDS) ${ }^{21}$ foi utilizada para avaliar níveis de desejabilidade social. A MC-SDS 
utiliza 33 afirmações para avaliar a tendência do participante a responder questionários de forma socialmente aceitável. O escore total da escala é obtido pela soma simples dos escores individuais. No presente estudo foi observado um alfa de Cronbach de 0,71.

Escolaridade, Status ocupacional e Status socioeconômico. Uma escala de sete níveis (ensino fundamental incompleto, 1 ; ensino fundamental completo, 2; ensino médio incompleto, 3 ; ensino médio completo, 4 ; ensino superior incompleto, 5; ensino superior completo, 6; pós-graduação, 7) foi utilizada para avaliar a escolaridade. Para avaliar o nível socioeconômico da amostra foi utilizado o Índice Quadrifatorial de Status Socioeconômico de Hollingshead (HI) ${ }^{13,22}$.

\section{Procedimento}

Integrantes do grupo de pesquisa, após receberem capacitação técnica para coleta de dados, entrevistaram as mães em sua própria residência, em dias e horários combinados com as mães. Um termo de consentimento livre e esclarecido, devidamente aprovado por um comitê de ética em pesquisa que autorizou a realização do presente estudo, foi utilizado para informar as mães sobre todos os detalhes relacionados à participação na pesquisa.

\section{Resultados e Discussão}

Análises foram conduzidas com o objetivo de verificar se o tamanho da amostra seria suficiente para garantir potência aos testes estatísticos. Em praticamente todos as análises a potência foi adequada $(\hat{a}>0,80)$. As distribuições univariadas e bivariadas de todas as variáveis consideradas no estudo foram examinadas com o objetivo de avaliar normalidade, homogeneidade de variância e presença de dados extremos. Apenas a medida de conhecimento do desenvolvimento infantil precisou sofrer transformação, que resolveu todos os problemas de simetria, curtose e heterogeneidade de variância. Um único caso de valor extremo univariado foi identificado e excluído das análises. As demais variáveis não apresentaram anomalias dignas de nota. Para clarificar a interpretação dos resultados, apresentamos as médias e os desvios-padrão das variáveis não transformadas. Utilizamos variáveis transformadas nas análises de correlação ${ }^{23}$.

A apresentação dos resultados e discussão foi organizada em duas partes. Na primeira foram discutidos aspectos descritivos e comparativos dos resultados obtidos nesta pesquisa. $\mathrm{Na}$ sequiência, foram examinadas correlações entre as variáveis estudadas.

Desejabilidade Social. Os escores relativos à desejabilidade social obtidos neste estudo $(M=$ $17,8, D P=4,57)$ mostraram-se muito semelhantes aos relatados em outros estudos ${ }^{12,24}$. As mães não apresentaram níveis de desejabilidade social superiores aos observados em outros estudos semelhantes ou que pudessem pôr em dúvida, sob esse aspecto, os resultados do estudo.

Conhecimento sobre o Desenvolvimento Infantil. As mães que participaram do estudo brasileiro acertaram em média $63 \%$ das questões do KIDI. Esse resultado indica um nível razoável de conhecimento do desenvolvimento infantil, que poderia, entretanto, ser expandido, por exemplo, por meio de cursos para pais.

Bornstein et al. ${ }^{25}$ conduziram um estudo com mães de sete nacionalidades (argentinas, belgas, francesas, israelenses, italianas, japonesas e norte-americanas) utilizando o KIDI. Entre outros aspectos, foram identificadas diferenças significativas no nível de conhecimento do desenvolvimento infantil entre mães de diferentes nacionalidades, mesmo quando outras variáveis (e.g., escolaridade) foram controladas. Os resultados obtidos com a aplicação do KIDI no presente estudo $(M=0,63, D P=0,11)$ não diferiram significativamente dos observados por Bornstein et al. ${ }^{25}$ na Argentina, e no Japão.

Autopercepções do papel parental. A Figura 1 apresenta os resultados obtidos com a aplicação do SPPR. Foi conduzida uma análise de variância para medidas repetidas com o objetivo de comparar os escores obtidos nas quatro subescalas do SPPR (Competência, Satisfação, Investimento e Integração). A análise revelou diferenças significativas entre os escores das subescalas, Lambda de Wilks $F(3,61)=$ $62,8, p<0,001$. O aspecto mais positivamente 
Figura 1 - Autopercepções maternas em quatro domínios (Satisfação, Investimento, Integração e Competência).

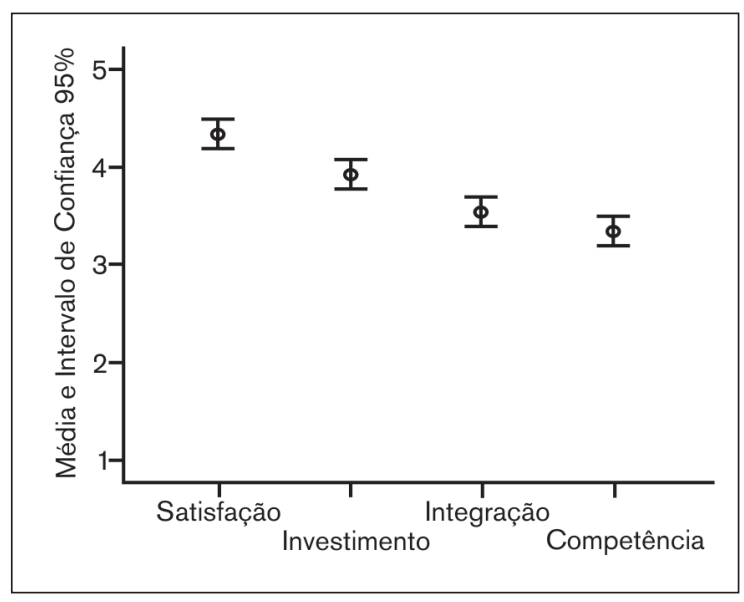

avaliado pelas mães foi a percepção da própria satisfação pessoal no desempenho do papel parental. Resultado semelhante a esse foi observado em estudo anteriores ${ }^{24,26}$. O segundo aspecto avaliado de forma mais positiva foi a percepção do próprio investimento no papel parental. Comparações post hoc revelaram que as avaliações das mães sobre sua satisfação e investimento foram significativamente mais positivas que suas avaliações sobre competência e integração de papeis.

Os resultados obtidos neste estudo com aplicação do SPPR foram comparados aos obtidos em um estudo realizado com mães norteamericanas de origem européia ${ }^{26}$. Testes $t$ revelaram que as percepções das mães deste estudo foram significativamente menos positivas que as das mães norte-americanas em todas as áreas investigadas, com exceção da percepção do próprio investimento. As mães brasileiras avaliaram o próprio investimento no papel parental mais positivamente que as norte-americanas. Nas comparações, o tamanho do efeito de Cohen variou de 0,61 a 1,31 .

Atribuições parentais de causalidade. A Figura 2 apresenta os resultados obtidos com a aplicação do PAQ. Foi conduzida uma análise de variância para medidas repetidas agora com o objetivo de comparar os escores obtidos pelas mães brasileiras nas quatro subescalas do PAQ (Atribuição Interna para o Sucesso, Atribuição Interna para o Fracasso, Atribuição Externa para
Figura 2 - Atribuições de causalidade para sucesso e fracasso em atividades parentais

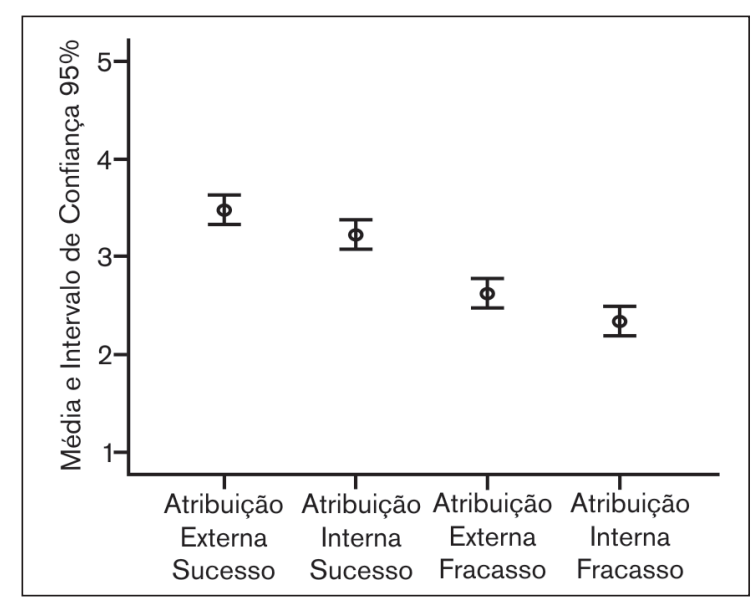

o Sucesso, Atribuição Externa para o Fracasso). A análise revelou diferenças significativas entre os escores das subescalas, Lambda de Wilks, $F$ $(3,62)=31,5, p<0,001$. De maneira geral, as mães tenderam a avaliar as causas externas como mais importantes do que as internas, tanto para o sucesso quanto para o fracasso nas atividades parentais.

Correlações. A Tabela 2 apresenta uma matriz correlação de Pearson de ordem zero envolvendo todas as variáveis consideradas neste estudo. A medida de desejabilidade social das mães não se mostrou significativamente associada a nenhuma das variáveis incluídas neste estudo. Dessa forma, para simplificação essa variável foi excluída da tabela e do restante da discussão.

Como se pode verificar na Tabela 2, a idade, a escolaridade e o status socioeconômico das mães correlacionaram-se significativamente entre si. Correlações bastante similares às observadas neste estudo vêm sendo sistematicamente reportadas na literatura ${ }^{12,27}$.

Conhecimento do Desenvolvimento Infantil. Corroborando estudos anteriores ${ }^{14}$, observou-se que mães com maior escolaridade e status econômico apresentaram um maior conhecimento do desenvolvimento infantil. O conhecimento do desenvolvimento infantil não apresentou correlação significativa com a idade das mães. Esse resultados são consistentes com estudos envolvendo exclusivamente mulheres $\operatorname{adultas}^{27}$. 
Tabela 2 - Correlações de ordem zero entre as variáveis estudadas

\begin{tabular}{|c|c|c|c|c|c|c|c|c|c|c|c|}
\hline Variável & 2 & 3 & 4 & 5 & 6 & 7 & 8 & 9 & 10 & 11 & 12 \\
\hline 1. Idade Mãe (anos) & $0,52^{\text {*** }}$ & $0,53^{\text {*** }}$ & 0,00 & 0,15 & $0,31^{*}$ & $0,36^{\text {*** }}$ & $0,47^{\text {*** }}$ & 0,22 & $-0,26^{*}$ & 0,03 & 0,01 \\
\hline 2. Escolaridade Mãe ${ }^{a}$ & - & $0,82^{\text {*** }}$ & $0,41^{\text {*** }}$ & $0,27^{*}$ & 0,24 & $0,31^{*}$ & $0,46^{* *}$ & $-0,02$ & $-0,05$ & 0,04 & 0,10 \\
\hline 3. Status Socioeconômico ${ }^{\mathrm{b}}$ & & - & $0,34^{* *}$ & 0,19 & $0,33^{* *}$ & 0,24 & $0,50^{* *}$ & 0,09 & $-0,09$ & 0,03 & 0,17 \\
\hline 4. Conhecimento do Desenvolvimento & & & - & $0,36^{\text {** }}$ & 0,20 & 0,17 & $0,32^{*}$ & 0,00 & $-0,09$ & $-0,13$ & $-0,04$ \\
\hline \multicolumn{12}{|l|}{ Autopercepções } \\
\hline 5. Investimento & & & & - & $0,26^{*}$ & 0,15 & $0,31^{*}$ & 0,02 & 0,02 & $-0,13$ & $-0,08$ \\
\hline 6. Integração & & & & & - & $0,34^{\text {*** }}$ & $0,44^{* *}$ & $0,34^{* *}$ & $-0,23$ & $-0,11$ & $-0,19$ \\
\hline 7. Competência & & & & & & - & $0,49^{* *}$ & 0,10 & $-0,27^{*}$ & 0,07 & $-0,33^{\text {*** }}$ \\
\hline 8. Satisfação & & & & & & & - & 0,05 & $-0,21$ & 0,01 & $-0,08$ \\
\hline \multicolumn{12}{|l|}{ Atribuição Externa } \\
\hline 9. Sucesso & & & & & & & & - & $-0,38^{* *}$ & 0,14 & $-0,09$ \\
\hline 10. Fracasso & & & & & & & & & - & $0,25^{*}$ & $0,53^{\text {*** }}$ \\
\hline \multicolumn{12}{|l|}{ Atribuição Interna } \\
\hline 11. Sucesso & & & & & & & & & & - & 0,24 \\
\hline 12. Fracasso & & & & & & & & & & & - \\
\hline
\end{tabular}

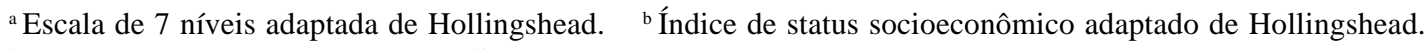

* Significativo para alfa $<0,05$. $\quad{ }^{* *}$ Significativo para alfa $<0,01$. (bilateral).

O conhecimento do desenvolvimento infantil mostrou-se positivamente correlacionado a duas das quatro medidas de autopercepção parental. Mães com maior conhecimento sobre o desenvolvimento infantil também apresentaram autopercepções mais positivas acerca da satisfação e do investimento parental. Esses resultados são coerentes com estudos já discutidos ${ }^{11,12}$. Por outro lado, o presente estudo não detectou correlações significativas entre conhecimento parental e atribuição de causalidade. Esse resultado diverge do apresentado por Stevens ${ }^{9}$, que identificou correlação inversa entre conhecimento e atribuições de causalidade externas.

Autopercepções parentais. Como se pode observar na Tabela 2, as escalas Competência, Satisfação e Integração correlacionaram-se entre si. Apenas as autopercepções de competência e investimento não se correlacionaram. Esses resultados são, em grande medida, similares aos apresentados em outros estudos ${ }^{12}$, indicando que as diferentes autopercepções parentais estão normalmente relacionadas entre si.

Conforme se pode observar na Tabela 2, várias das medidas de autopercepção parental apresentaram correlações significativas com a idade, a escolaridade e o status socioeconômico das mães. Em geral, mães mais velhas e com mais escolaridade apresentaram uma autopercepção mais positiva de seu próprio desempenho do papel parental. Esses resultados são similares aos obtidos por Bornstein et al. ${ }^{26}$.

Três correlações significativas entre autopercepções e atribuições de causalidade foram identificadas. A autopercepção parental da competência apresentou relação inversa com as atribuições internas e externas de fracasso. Pode-se dizer que esses resultados são coerentes. Mães que se perceberam mais competentes avaliaram que as causas apresentadas, quer tenham sido externas (e.g., comportamento da criança) ou internas (e.g., esforço), menos freqüentemente eram responsáveis por seu fracasso no desempenho do papel parental. A autopercepção parental da integração correlacionou-se positivamente à atribuição externa para o sucesso. Mães que avaliaram mais positivamente o equilíbrio dos diferentes papéis parentais avaliaram que as causas externas seriam mais importantes para o seu desempenho do papel parental.

Atribuições parentais de causalidade. As atribuições de causalidade parentais não se correlacionaram a nenhuma das variáveis socioeconômicas e demográficas consideradas neste estudo. Trata-se de uma diferença marcante em relação às outras cognições investigadas. Esses 
resultados parecem ser coerentes com algumas posições teóricas e evidências empíricas relacionadas ao lócus de controle parental ${ }^{7}$. Estilos de atribuição de causalidade seriam adquiridos pelos indivíduos ao longo de suas vidas e estariam mais relacionados com tipos de experiências que guardariam pouca relação com a escolaridade e o status socioeconômico. Por exemplo, evidências indicam que a experiência de ter bebês com níveis de cólica importantes pode promover a percepção de um lócus de controle externo nas mães, quando estas avaliam que sua capacidade de controlar as cólicas é reduzida. A percepção de lócus de controle externo, por sua vez, produziria uma redução da percepção da própria competência ${ }^{28}$.

\section{CONCLUSÕES}

As cognições parentais são de vital importância para o entendimento da psicologia parental e do próprio desenvolvimento infantil. $\mathrm{O}$ aumento da quantidade de pesquisas sobre a cognição parental está diretamente relacionado ao crescente reconhecimento dessa importância. O número relativamente pequeno de pesquisas brasileiras acerca das cognições parentais, envolvendo a utilização de instrumentos de medida validados empiricamente, reforçou a avaliação de que mais pesquisas sobre esse assunto deveriam ser conduzidas no país.

O presente trabalho ofereceu inicialmente uma breve revisão de evidências empíricas referentes a três tipos de cognições parentais: conhecimentos sobre o desenvolvimento, atribuições de causalidade e autopercepções parentais. A importância dessas cognições para a psicologia parental, assim como evidências acerca de suas múltiplas associações, foram discutidas.

Deve-se ressaltar que o presente estudo teve um caráter eminentemente exploratório, até porque evidências acerca dessas cognições envolvendo mães brasileiras ainda são bastante escassas. Não foi possível identificar, por exemplo, estudos nacionais que tivessem investigado simultaneamente todas as cognições parentais aqui consideradas. Assim, as diferenças e semelhanças encontradas não foram analisadas a partir de mo- delos teóricos específicos. Assim como não foram previamente definidas e testadas hipóteses específicas acerca dessas cognições.

Os resultados da presente pesquisa permitiram ampliar nosso conhecimento sobre cognições de mães brasileiras a respeito de temas relacionados à maternidade e ao desenvolvimento humano. Além de oferecer resultados descritivos, foi possível situar esses resultados tendo em vista evidências produzidas em estudos nacionais e internacionais anteriores. De uma maneira geral essas comparações permitiram concluir que os resultados obtidos foram, em larga medida, compatíveis com diversos estudos anteriores. Por outro lado, foi possível identificar a existência de variações transculturais ou transnacionais importantes. Explicações para essas semelhanças e diferenças deverão ser exploradas em novos estudos.

O nível de conhecimento sobre o desenvolvimento infantil apresentado pelas mães brasileiras mostrou-se similar, por exemplo, ao observado em um estudo com mães argentinas. Considerando os resultados obtidos, pode-se dizer que o conhecimento das mães pode ser ampliado. Corroborando estudos anteriores, as mães apresentaram uma percepção positiva sobre si mesmas, com destaque para percepção da própria satisfação pessoal no desempenho do papel parental e do investimento no papel parental.

O presente estudo forneceu ainda informações sobre como as cognições maternas estão associadas entre si e como essas cognições se correlacionam com outras variáveis que consideramos relevantes, como o nível educacional. Foram identificadas diversas associações entre o nível de escolaridade, o conhecimento sobre o desenvolvimento infantil, autopercepções parentais e atribuições de causalidade parentais. De fato, deve-se destacar que os padrões de associação observados neste estudo foram largamente similares aos obtidos em outros estudos nacionais e internacionais. Esses resultados lançam luz sobre as cognições parentais como um sistema de cognições e poderão contribuir para a construção de modelos explicativos integrados das cognições parentais. Novos estudos dos autores estão sendo encaminhados nesse sentido.

Obviamente, o trabalho teve também algumas limitações. Os instrumentos utilizados 
neste estudo foram cuidadosamente estudados, selecionados e previamente testados em estudos anteriores. Apesar disso, alguns coeficientes de fidedignidade se revelaram abaixo do desejado. Adicionalmente, deve-se mencionar que nenhum dos instrumentos utilizados avaliou valores parentais e o suporte social. Pretende-se incluir em próximas investigações medidas de valores parentais (e.g., metas de socialização) e suporte social (e.g., nível de suporte informal e institucional) sobre a psicologia parental. A participação dos pais na criação dos filhos em países industrializados e mesmo no Brasil vem crescendo significativamente. Pais têm assumido um papel cada vez mais importante e dedicado mais horas aos cuidados de seus filhos. Desta forma, entende-se que futuros estudos devem envolver não apenas mães, mas também pais.

Este trabalho envolveu pesquisadores de diferentes instituições nacionais e estrangeiras. Essa colaboração, apesar de complexa, mostrouse fecunda e mutuamente interessante. Vale ainda mencionar que estudos com amostras de diferentes grupos dentro de uma mesma cultura são pouco freqüentes na literatura e permitem o conhecimento de diferenças intraculturais. Uma iniciativa nesse sentido foi realizada recentemente num estudo que envolveu mães residentes em seis estados brasileiros ${ }^{14}$.

\begin{abstract}
The present study investigated three types of parental cognitions (parenting knowledge, parents' self-perceptions, causal attributions in parenting) and had three objectives: 1) to enlarge our knowledge about Brazilian mothers' cognitions concerning parenting; 2) to compare findings produced in the present study with findings produced in previous studies; and 3) to investigate associations between mothers' cognitions and between mothers' cognitions and certain sociodemographic variables. Sixty-six primiparous mothers, aged 18 or older, from intact families, living in the city of Rio de Janeiro, participated in the study. Mothers completed Brazilian versions of four instruments (Knowledge of Infant Development Inventory, SelfPerceptions of the Parental Role Instrument, The Parent Attributions Questionnaire, MarloweCrowne Social Desirability Scale) and a sociodemographic questionnaire. The mothers answered correctly about $63 \%$ of the questions of the instrument that evaluated parenting knowledge, indicating that their knowledge about child development can be enlarged. Concerning parental self-perceptions mothers evaluated more positively their satisfaction and investment in the parental role. The mothers tended to evaluate external causes (e.g., child behavior) as more important than the internal ones (e.g., effort) for the success and failure in parental activities. Several associations between mothers' cognitions and between mothers' cognitions and sociodemographic variables (e.g., educational attainment) were identified. The results were, to a great extent, consistent with previous national and international studies and can contribute to our understanding of parental cognitions as a complex system.
\end{abstract}

Key words: Parental cognitions. Parenting knowledge. Self-perceptions. Causal attributions.

\section{REFERENCIAS}

1. Bornstein MH. Specificity of mother-infant interaction: the influence of maternal age, employment status, and parenthood status [protocolo de pesquisa 88-CH-32]. Bethesda: NICHD; 2000.

2. Seidl de Moura ML. Interação mãe-bebê e desenvolvimento infantil: um estudo longitudinal e transcultural [projeto de pesquisa apresentado ao CNPq]. Rio de Janeiro: Universidade do Estado do Rio de Janeiro; 1999.
3. Sigel IE, McGillicuddy De Lisi AV. Parent beliefs are cognitions: the dynamic belief systems model. In: Bornstein MH, editor. Handbook of parenting. $2^{\text {nd }}$ ed. Mahwah (NJ): Lawrence Erlbaum; 2002. v. 3, p. 485-508.

4. Goodnow JJ. Parents' knowledge and expectations. In: Bornstein $\mathrm{MH}$, editor. Handbook of parenting. Mahwah (NJ): Lawrence Erlbaum; 1995. p. 305-32.

5. Benasich AA, Brooks-Gunn J. Maternal attitudes and knowledge of child-rearing: associations with family and child outcomes. Child Dev. 1996;67(3):1186-205. 
6. Angelillo I, Ricciardi G, Rossi P, Pantisano P, Langiano E, Pavia M. Mothers and vaccination: knowledge, attitudes, and behaviour in Italy. Bull World Health Organ. 1999;77:224-9.

7. Coleman PK, Karraker KH. Self-efficacy and parenting quality: findings and future applications. Dev Rev. 1997;18:47-85.

8. Bugental DB, Blue J, Cruzcosa M. Perceived control over caregiving outcomes: implications for child abuse. Dev Psychol. 1989;25:532-9.

9. Stevens JH. Shared knowledge about infants among fathers and mothers. J Genet Psychol. 1988;149:515-25.

10. Bandura A. Human agency in social cognitive theory. Am Psychol. 1989;44:1175-84.

11. Ruchala P, James DC. Social support, knowledge of infant development, and maternal confidence among adolescent and adult mothers. J Obstet Gynecol Neonatal Nurs. 1997;26:685-9.

12. Ribas AFP, Ribas Jr RC, Valente AA. Bemestar emocional de mães e pais e o exercício do papel parental: uma investigação empírica. Rev Bras Crescimento Desenvolv Hum. 2006;16(3):28-38. o

13. Ribas Jr RC, Seidl de Moura ML, Gomes AAN, Soares ID, Bornstein MH.

Socioeconomic status in brazilian psychological research: validity, measurement, and application. Estud Psicol (Natal). 2003;8:375-83.

14. Seidl de Moura ML, Ribas Jr RC, Piccinini CA, Bastos ACS, Magalhães CMC, Vieira $\mathrm{ML}$, et al. Conhecimento sobre desenvolvimento infantil em mães primíparas de diferentes centros urbanos do Brasil. Estud Psicol (Natal). 2004;9:421-30.

15. Rabinovich EP, Coser ACPH, Travaglini D, Esteves EN. A comparative study of naming children from two socioeconomic groups. J Soc Psychol. 1994;134:553-5.

16. Brito Dias CMS, Tróccoli BT. Lócus de controle materno, satisfação com a vida e relacionamento com o filho adolescente. Psicol Teor Pesqui. 1999;15:209-18.

17. MacPhee D. Manual: knowledge of infant development inventory. University of North Carolina; 1981.

18. MacPhee D, Benson JB, Bullock D. Influences on maternal self-perceptions. In: Paper presented at 5th Biennial International Conference on Infant Studies; 1986 April 1013; Los Angeles, CA, USA

19 Ribas Jr RC. Cognições de mães brasileiras acerca da maternidade e do desenvolvimento humano: uma contribuição ao estudo da psicologia parental [tese]. Rio de Janeiro: Instituto de Psicologia da Universidade do Estado do Rio de Janeiro; 2004.

20. Sirignano SW, Lachman ME. Personality change during the transition to parenthood: the role of perceived infant temperament. Dev Psychol. 1985;21:558-67.

21. Ribas Jr RC, Seidl de Moura ML, Hutz CS. Adaptação brasileira da Escala de Desejabilidade Social de Marlowe-Crowne. Aval Psicol. 2004;3:83-91.

22. Hollingshead AB. Four factor index of social status. Department of Sociology of Yale University; 1975.

23. Tabachinick BG, Fidell LS. Using multivariate statistics. New York: Harper Collins; 1996.

24. Bornstein MH, Haynes O, Azuma H, Galperin C, Maital S, Ogino M, et al. A cross-national study of self-evaluations and attributions in parenting: Argentina, Belgium, France, Israel, Italy, Japan, and the United States. Dev Psychol. 1998;34:662-76.

25. Bornstein MH, Hahn CS, Suizzo MA, Haynes OM. Mothers' knowledge about child development and child-rearing: national and cross-national studies. Bethesda (MD): National Institute of Child Health and Human Development; 2006.

26. Bornstein MH, Hendricks C, Hahn CS, Haynes OM, Painter KM, Tamis-LeMonda CS. Contributors to self-perceived competence, satisfaction, investment, and role balance in maternal parenting: a multivariate ecological analysis. Parent Sci Pract. 2003;3:285-326.

27. Ribas Jr RC, Bornstein MH. Parenting knowledge: similarities and differences in Brazilian mothers and fathers. Int J Psychol. 2005;39:5-12.

28. Stifter C, Bono M. The effect of infant colic on maternal self-perceptions and mother-infant attachment. Child Care Health Dev. 1998;24:339-51. 